\title{
Spreading of Bubbles after Contacting the Lower Side of an Aerophilic Slide Immersed in Water
}

\author{
Hélène de Maleprade, Christophe Clanet, and David Quéré \\ Physique and Mécanique des Milieux Hétérogènes, UMR 7636 du CNRS, ESPCI, 75005 Paris, France \\ and LadHyX, UMR 7646 du CNRS, École Polytechnique, 91128 Palaiseau, France
}

(Received 15 February 2016; revised manuscript received 8 July 2016; published 25 August 2016)

\begin{abstract}
While the dynamics of complete wetting has been widely studied for liquids, the way a gas spreads on a solid is by far less known. We report here the events following the rise of a millimeter-size air bubble towards a textured material immersed in water and covered by a thin plastron of air. Bubbles contact the material either directly at the end of the rise, or after a few rebounds, which affects the initial shape of the bubble and the resulting dynamics of contact. Then, air spreads on the material, owing to surface tension and later buoyance, which tends to flatten further the bubble. The corresponding dynamics are shown to result from the inertial resistance of water, which explains how spreading bubbles reach centimeter sizes in typically $10 \mathrm{~ms}$.
\end{abstract}

DOI: 10.1103/PhysRevLett.117.094501

How liquids spread on wettable solids has been a major topic of interfacial hydrodynamics since the 1980s [1]. Drops whose flattening minimizes surface energy are first driven by surface tension $\gamma$ [2]; above the capillary length $a$, spreading results from gravity, since the liquid density $\rho$ is larger than that of surrounding air [3]. Resistance to motion has also been extensively discussed [4]. Friction at short scale is dominated by the dissipation close to the moving contact line, where viscous effects are enhanced [5]. Balancing this "line friction" with surface tension leads to Tanner's law: $r(t) \sim\left(\gamma \Omega^{3} t / \eta\right)^{1 / 10}$, where $\Omega$ is the drop volume and $\eta$ the liquid viscosity [2]. At large scale, line friction is overcome by bulk friction whose balance with gravity leads to Huppert's law: $r(t) \sim\left(\rho g \Omega^{3} t / \eta\right)^{1 / 8}$ [3]. The crossover between both regimes occurs around $a$, above which gravity dominates surface tension.

Here we consider bubbles spreading on aerophilic solids [Figs. 1(a) and 1(b)]. This can be seen as the complementary problem of wetting drops, where the two fluid phases have been exchanged. Bubble spreading is achieved using superhydrophobic materials [6], which are "wet" by air once immersed in water [7]. This property is exploited by insects and even mammals hiding or hunting underwater, and observed to be covered by a persistent plastron of air, serving as an oxygen reservoir or thermal insulator [8-10]. Wang et al. looked at the impact of bubbles on submerged lotus leaves and reported a rapid spreading of air, with a characteristic time of about $10 \mathrm{msec}$ [11], smaller by far than that of water wetting solids, on order of minutes to hours at centimetric scales [3]. Our aim is to quantify the dynamics of bubble spreading, and to understand its physics.

Our surfaces are made by dipping centimetric glass slides into a solution of silanized silica nanobeads with diameter $30 \mathrm{~nm}$ (Glaco, Soft99). After solvent evaporation, the coating is consolidated at $250{ }^{\circ} \mathrm{C}$ for $30 \mathrm{~min}$. Surfaces are transparent with a typical roughness $\Delta z=100 \mathrm{~nm}$ imaged by AFM in Figs. 1(c) and 1(d). These slides are aerophilic: once immersed at speed $V$ in water, they are always coated by a plastron of air $[12,13]$. At large $V$, air thickness $\varepsilon$ is given by the Landau-Levich law $\varepsilon \approx a\left(\eta_{a} V / \gamma\right)^{2 / 3}$ [14], with $\eta_{a}$ the air viscosity. For $V \approx 10 \mathrm{~mm} / \mathrm{s}$, we have $\varepsilon \approx 100 \mathrm{~nm}$, that is, the characteristic roughness $\Delta z$ of the solid. This thickness just corresponds to the filling of cavities by air: if we immerse the slide at $V=1-10 \mathrm{~mm} / \mathrm{s}, \varepsilon$ plateaus at the value $\varepsilon=\Delta z$ [15], which yields a film of reproducible thickness.

Slide sides sit on solid blocks adjusted to provide horizontality. Air bubbles with radius $R$ between 0.92 and $1.87 \mathrm{~mm}$ and released from calibrated needles located $3 \mathrm{~mm}$ to $1 \mathrm{~cm}$ beneath the slide rise at a velocity ranging from 20 to $30 \mathrm{~cm} / \mathrm{s}$ until they reach the plate. The water
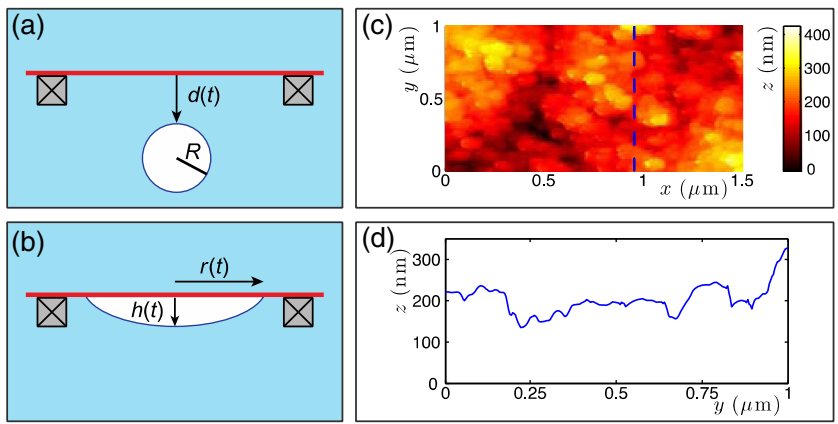

FIG. 1. Experimental setup and materials. (a) An air bubble of radius $R$ approaches a flat horizontal aerophilic slide (in red) immersed in water and covered by a thin film of air. (b) After contact, spontaneous spreading occurs and the bubble is characterized by its height $h(t)$ and radius $r(t)$. (c) AFM image in the plane $(x, y)$ of the surface showing its nanometric textures. (d) AFM profile along the dashed line in (c): the typical variation $\Delta z$ of the height $z(x, y)$ is $\Delta z \approx 100 \mathrm{~nm}$. 
tank is cubic with straight walls to avoid optical aberrations, and filled with deionized water. Observations are made with a high-speed video camera (Phantom Miro M310) at $23000 \mathrm{fps}$ (frames per second). Snapshots in Figs. 2(a) and 2(b) show that rising bubbles are dynamically flattened $[16,17]$. At the plate, either they bounce back [Fig. 2(a) and blue data in Figs. 2(c) and 2(d)] and eventually adopt a spherical shape, or they stop with a flattened shape at the surface, which they contact after a short delay [Fig. 2(b) and pink data in Figs. 2(c) and 2(d)]. Statistics over 100 bubbles indicate that the bouncing probability falls from $100 \%$ for hydrophilic and hydrophobic solids to $40 \%$ for our aerophilic plates [Fig. 2(c)]. Contact [plain circle in Fig. 2(d)] naturally occurs earlier when there is no rebound.

Bubbles generally bounce when they meet solids $[18,19]$, due to the lubrication pressure in the film as water is evacuated [20,21]. The rebounds are soft [Fig. 2(d), blue data], so that bubbles slow down and eventually reach a spherical shape, as seen in Fig. 2(a). However, the thin film of air on the material can induce slip, as water is squeezed by the rising bubble. Owing to this efficient drainage, the bubble can stop without bouncing and keep its dynamic shape at contact [Fig. 2(b) and pink data in Figs. 2(c) and 2(d)]. We successively consider these two initial situations. We first discuss the case of flattened bubbles, and Fig. 3 shows for five similar experiments how the bubble-plate radius $r$ grows as a function of time $t$, whose origin is taken at the first moment of contact.

We can identify three dynamical stages in Fig. 3, as stressed by colors. The first regime (in green) concerns very short times $(t<0.2 \mathrm{~ms})$. It is shown in Fig. 3(a) where movies are shot from the top through the transparent plate, at a rate of $70000 \mathrm{fps}$. The contact $(r<R)$ propagates without modifying the global shape of the bubble. In this regime, its radius $r$ increases linearly with time $t$ as highlighted by the line of slope 1 drawn in Fig. 3(d). Water between the flat bubble and the plate is squeezed between air and an aerophilic material coated by air. The contact expands as this water film of thickness $\delta$ retracts, which generates the rim seen in Fig. 3(a). As modeled by Taylor [22] and Culick [23], the balance of surface tension $2 \gamma$ with inertia $\rho \delta V^{2}$ yields a constant retraction velocity $V=(2 \gamma / \rho \delta)^{1 / 2}$. Hence a linear growth for the contact

$$
r(t)=\left(\frac{2 \gamma}{\rho \delta}\right)^{1 / 2} t
$$

Experiments in this regime provide reproducible velocities of $4.0 \pm 0.4 \mathrm{~m} / \mathrm{s}$, which, using Eq. (1), implies a film thickness $\delta=9 \pm 2 \mu \mathrm{m}$. Measuring water thickness from macrophotos, we get on ten experiments $\delta=15 \pm 5 \mu \mathrm{m}$, close to the expected value.

At a time $t \approx R(\rho \delta / 2 \gamma)^{1 / 2} \approx 0.3 \mathrm{~ms}$, the scale $R$ of the bubble separates the regime of contact $(r<R)$ from spreading in the true sense $(r>R)$. Then a second regime (marked in red) takes place and the bubble is observed in Figs. 3(c) and 3(d) to expand as $t^{\alpha}$, with $\alpha=0.35 \pm 0.04$. As for spreading drops, we assume that motion is driven by surface tension that tends to flatten the water-air interface [Fig. 3(b)], whose curvature scales as $h / r^{2}$, denoting the bubble radius and height as $r$ and $h$. The expanding bubble of volume $\Omega$ displaces a volume of water comparable to $\Omega$ at a typical velocity $V=d r / d t$ of $1 \mathrm{~m} / \mathrm{s}$ [Fig. 3(d)]. The corresponding Reynolds number $\rho R V / \eta$ is $10^{3}$, much larger than unity. Therefore, we assume that the dominant resistance is water inertia. Using volume conservation $\Omega \sim h r^{2}$, the balance of dynamical pressure $\rho V^{2}$ with Laplace pressure $\gamma h / r^{2}$ leads to

$$
r(t) \approx\left(\frac{\gamma \Omega}{\rho}\right)^{1 / 6} t^{1 / 3}
$$

This inertiocapillary regime is markedly different from Tanner's dynamics in $t^{1 / 10}$ [2]. The exponent $1 / 3$ drawn with a red line in Fig. 3(d) is in good agreement with the data. Equation (2) predicts a typical expansion of $1 \mathrm{~mm}$ in $1 \mathrm{~ms}$ for a millimetric bubble, again in accordance with observations. In the case of partial wetting, we expect
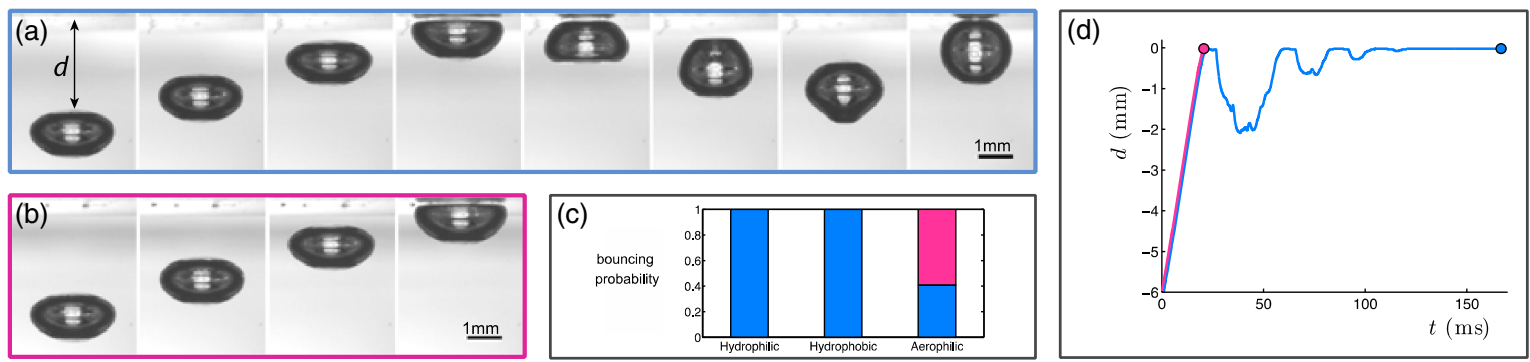

FIG. 2. Bubble dynamics before contacting an aerophilic plate, with $R=1 \mathrm{~mm}$. (a) Either bubbles bounce off the surface (b) or stop without rebound. Images are separated by $3.1 \mathrm{~ms}$ and the final picture in each set is the last frame before contact [delayed in (a) due to bouncing]. Corresponding movies are movies 1 and 2. (c) Probability of bouncing (in blue) against hydrophilic, hydrophobic, or aerophilic plates. (d) Bubble depth $d$ as a function of time $t$ : the blue and pink curves show typical trajectories for bouncing and nonbouncing bubbles, respectively. Plain circles indicate the last moment before contact with the plate. 

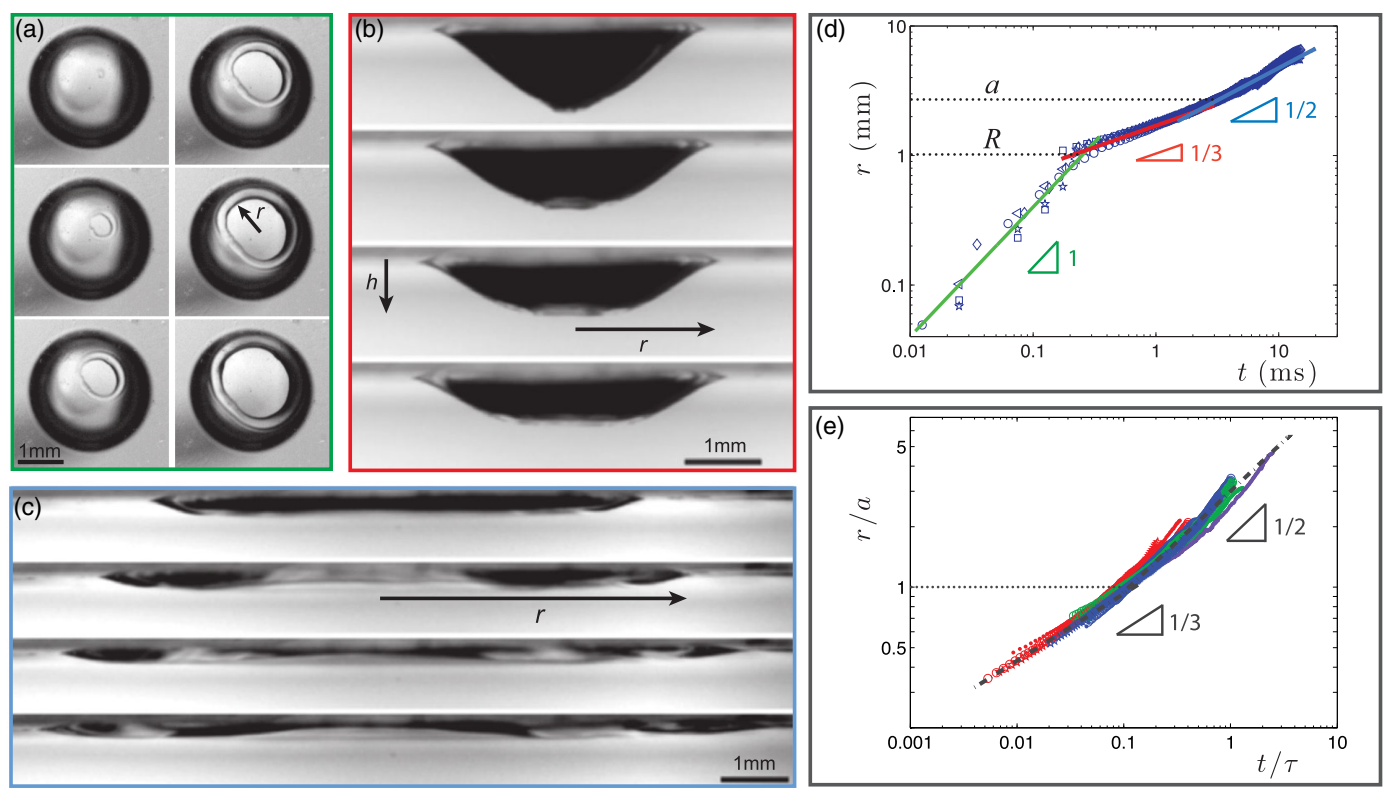

FIG. 3. Spreading of a flattened bubble. (a) Top view of the propagation of contact (movie 3); interval between images is $\Delta t=0.1 \mathrm{~ms}$. (b) In the following regime of spreading (seen from the side for $t>1.4 \mathrm{~ms}$ and $\Delta t=0.21 \mathrm{~ms}$ ), the contact radius $r$ expands beyond the radius $R$ of the initial bubble. (c) Later $(t>7.5 \mathrm{~ms}$ and $\Delta t=1.7 \mathrm{~ms}$ ), the bubble spreads in a much flatter configuration. Movie 4 corresponds to $\mathrm{b}$ and c. (d) Contact size $r$ as a function of time $t$ for $R=1 \mathrm{~mm}$, for five similar experiments (noted with different symbols). In this log-log plot, $r$ obeys three successive scaling laws of exponents $1,1 / 3$ and $1 / 2$ (green, red, and blue lines). (e) Dimensionless contact radius $r / a$ as a function of time $t$ normalized by $\tau=a^{2} /(\Omega g)^{1 / 2}$. Colors and symbols respectively stand for bubble size $[R=1.0$ (red); 1.3 (blue); 1.5 (green); $1.9 \mathrm{~mm}$ (purple)] and water viscosity $[\eta=1$ (dots); $\eta=2$ (circles); $\eta=3 \mathrm{mPas}$ (stars)].

bubbles to stop at a radius comparable to their size, so that spreading should only obey Eq. (2), as indeed shown in the Supplemental Material [24].

The dynamics in Fig. 3(d) bends up when the radius becomes $2-3 \mathrm{~mm}$. This corresponds to a change in bubble shape from curved [Fig. 3(b)] to flat [Fig. 3(c)]. For $r>a=(\gamma / \rho g)^{1 / 2}(2.5 \mathrm{~mm}$ for water), hydrostatic pressure $\rho g h$ overcomes Laplace pressure $\gamma h / r^{2}$, and it drives spreading. At such scale and $d r / d t \sim 1 \mathrm{~m} / \mathrm{s}$, motion remains resisted by water inertia, and the new pressure balance, $\rho V^{2} \sim \rho g h$, yields

$$
r(t) \approx(g \Omega)^{1 / 4} t^{1 / 2}
$$

In this inertiogravitational regime, bubble dynamics also differs from that of viscous puddles in $t^{1 / 8}$ [3]. Spreading still obeys a scaling law, whose exponent $1 / 2$ fits data in a satisfactory way [blue line in Fig. 3(d)] and reflects the inflection of the dynamics beyond $r \approx a$, at the crossover between Eqs. (2) and (3).

We tested the universality of the spreading laws by varying the volume $\Omega$ and viscosity $\eta$ (by adding glycerol to water). In Fig. 3(e), the contact radius $r$ normalized by the capillary length $a$ is plotted as a function of time $t$ scaled by $\tau=a^{2} /(g \Omega)^{1 / 2}$. In both regimes, the successive scaling laws are close to that predicted by Eqs. (2) $\left(r / a \approx(t / \tau)^{1 / 3}\right)$ and (3) $\left(r / a \approx(t / \tau)^{1 / 2}\right)$. Numerical coefficients deduced from fits are of order unity, with respective values of $2.1 \pm$ 0.1 and $3.1 \pm 0.4$. In the gravitational regime, the presence of waves at the bubble surface (usual in inertial regimes) might slightly affect the dynamics of the smallest bubbles.

As stressed earlier, globules at contact can also be spherical [Fig. 2(a)]. This initial difference should not modify the spreading stage $(r>R)$, but it necessarily affects the short-time dynamics, since water between a spherical bubble and a flat solid does not have a constant thickness. As shown in Fig. 4(b), the whole dynamics now looks unique and characterized by a slope $1 / 2$, with a regime of contact $(r<R$, in green) indeed modified compared to the previous case [Eq. (1)]. This can be understood by considering that the thickness $\delta$ in Culick's law $\left[d r / d t=(2 \gamma / \rho \delta)^{1 / 2}\right]$ depends on $r$. For a sphere-plane geometry, $\delta$ scales as $r^{2} / R$, which yields

$$
r(t) \approx\left(\frac{\gamma R}{\rho}\right)^{1 / 4} t^{1 / 2}
$$

The dynamics is close to that of spherical bubbles merging in water reported by Paulsen et al. [25]. Munro et al. provide an analytical expression for the growth of contact between such bubbles [26]. The contact size obeys the scaling of Eq. (4) with a numerical coefficient 

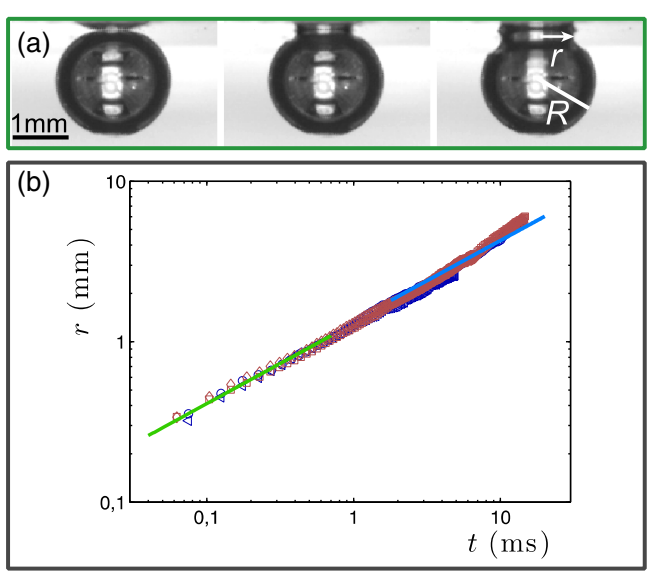

FIG. 4. (a) Side view of the contact propagation between a spherical bubble $(R=0.97 \mathrm{~mm})$ and the plate, with $\Delta t=$ $0.16 \mathrm{~ms}$ (movie 5). (b) Contact radius $r$ as a function of $t$. Different symbols correspond to different experiments (showing the reproducibility of the dynamics), with a bubble made of air (blue) or neon (brown). Green and blue lines both have a slope $1 / 2$.

$(32 / 3)^{1 / 4} \approx 1.8$, as calculated by Munro et al. The coefficient deduced from the fit in Fig. 4(b) is $2.5 \pm 0.1$, slightly larger than 1.8: there is less water to evacuate in a bubbleplate geometry, and smaller inertia leads to faster dynamics. The average radius is $R_{\mathrm{av}}=2 R$, which induces an additional factor of $2^{1 / 4}$ compared to the case of two spheres of radius $R$. The corresponding coefficient is $(64 / 3)^{1 / 4} \approx$ 2.15 , in closer agreement to our data.

Once contact is established $(r \approx R)$, spreading should follow the laws derived earlier. But the first and third regimes [Eqs. (4) and (3)] now both scale as $t^{1 / 2}$, as indeed seen in Fig. 4(b). A capillary regime of slightly smaller exponent [that is, 1/3, see Eq. (2)] can only match the regimes in $t^{1 / 2}$ if the coefficient of $t^{1 / 2}$ is larger in Eq. (4) than in Eq. (3). Since $\Omega$ scales as $R^{3}$, the ratio between these two coefficients reduces to $(a / R)^{1 / 2}$. This quantity of order unity slowly varies with the bubble size, which explains the quasicontinuity of data in Fig. 4(b). We notice that a capillary regime in $t^{1 / 3}$ cannot match the first and third regimes in $t^{1 / 2}$ if $(a / R)^{1 / 2}$ is smaller than unity. Selfconsistently, bubbles with $R>a$ are deformed by gravity and cannot be quasi-spherical at contact. The spreading dynamics of a larger bubble deformed by gravity after bouncing, hence having a flattened shape before contact, is shown in Ref. [24] to bring us back to the successive regimes observed in Fig. 3.

Spreading drops and bubbles have common features and marked differences. In both cases, liquid-air interfaces deform and flatten under the action of surface tension and gravity. A cascade of scaling laws is observed, but exponents are found to be much larger for bubbles, which is attributed to the prevalence of inertia over viscosity. For bubbles, viscous resistance might exist both inside the displaced liquid and in the wedge of air progressing at a velocity $V$. In the wedge of dynamic contact angle $\theta$, the Huh-Scriven "line friction" integrated over a bubble of size $R$ scales as $\eta_{a} V R / \theta$ [5]. If we assume Tanner's law for the contact angle, that is, $\theta \approx\left(\eta_{a} V / \gamma\right)^{1 / 3}$, we can construct a special Reynolds number comparing liquid inertia and wedge dissipation. It can be written $\mathrm{Re}=\mathrm{WeCa}^{-2 / 3}$, where $\mathrm{We}=\rho R V^{2} / \gamma$ and $\mathrm{Ca}=\eta_{a} V / \gamma$ are the Weber number in water and capillary number in air. For a velocity of $1 \mathrm{~m} / \mathrm{s}$, these numbers are 10 and $10^{-3}$ and Re is about $10^{3}$, much larger than unity. As shown in Fig. 4(b) (and the Supplemental Material [24]), a bubble made of neon 1.6 times more viscous than air indeed spreads the same way. Viscous dissipation also takes place inside the liquid, and its comparison with inertia results in a "usual" Reynolds number $\operatorname{Re}=\rho R V / \eta \approx 10^{3} \gg 1$. Hence bubbles spreading in liquids of viscosity $\eta=1,2$ and $3 \mathrm{mPa}$ s have undistinguishable dynamics [Fig. 3(d) and Supplemental Material [24]].

Bubbles in water wetting an aerophilic plate have a characteristic spreading time $\tau$ much shorter than in usual spreading. This time was found to scale as $a^{2} /(g \Omega)^{1 / 2}$, a quantity on the order of $10 \mathrm{~ms}$, in agreement with our observations [Figs. 3(d) and 4(b)] and with that by Wang et al. for air spreading on immersed lotus [11]. It would be interesting to see how spreading is affected when largely increasing the viscosity of the surrounding liquid. Also, the capillary regime in water can only hold between $R$ and $a$, that is, 1 and $2-3 \mathrm{~mm}$. It would be worth testing this regime in microgravity, where we do not expect buoyance to limit it.

We thank Julien Dupré de Baubigny for his help on AFM images, and Étienne Reyssat for his valuable comments on the manuscript.

[1] P.-G. de Gennes, Wetting: statics and dynamics, Rev. Mod. Phys. 57, 827 (1985).

[2] L.H. Tanner, The spreading of silicon oil drops on horizontal surfaces, J. Phys. D 12, 1473 (1979).

[3] H. E. Huppert, The propagation of two-dimensional and axisymmetric viscous gravity currents over a rigid horizontal surface, J. Fluid Mech. 121, 43 (1982).

[4] J.H. Snoeijer and B. Andreotti, Moving contact lines: Scales, regimes, and dynamical transitions, Annu. Rev. Fluid Mech. 45, 269 (2013).

[5] C. Huh and L. E. Scriven, Hydrodynamic model of steady movement of a solid/liquid/fluid contact line, J. Colloid Interface Sci. 35, 85 (1971).

[6] R. Blossey, Self-cleaning surfaces-virtual realities, Nat. Mater. 2, 301 (2003).

[7] C. Shi, X. Cui, X. Zhang, P. Tchoukov, Q. Liu, N. Encinas, M. Paven, F. Geyer, D. Vollmer, Z. Xu, H.-J. Butt, and H. Zeng, Interaction between air bubbles and superhydrophobic surfaces in aqueous solutions, Langmuir 31, 7317 (2015). 
[8] A. Balmert, H. F. Bohn, P. Ditsche-Kuru, and W. Barthlott, Dry under water: Comparative morphology and functional aspects of air-retaining insect surfaces, J. Morphol. 272, 442 (2011).

[9] M. R. Flynn and J. W. M. Bush, Underwater breathing: the mechanics of plastron respiration, J. Fluid Mech. 608, 275 (2008).

[10] W. A. Calder, Temperature relations and underwater endurance of the smallest homeothermic diver, the water shrew, Comp. Biochem. Physiol. 30, 1075 (1969).

[11] J. Wang, Y. Zeng, F. Q. Nie, J. Zhai, and L. Jiang, Air bubble bursting effect of lotus leaf, Langmuir 25, 14129 (2009).

[12] P. R. Jones, X. Hao, E. R. Cruz-Chu, K. Rykaczewski, K. Nandy, T. M. Schutzius, K. K. Varanasi, C. M. Megaridis, J. H. Walther, P. Koumoutsakos, H. D. Espinosa, and N. A. Patankar, Sustaining dry surfaces under water, Sci. Rep. 5, 12311 (2015).

[13] A. Marmur, Underwater superhydrophobicity: Theoretical feasibility, Langmuir 22, 1400 (2006).

[14] L. Landau and V. Levich, Dragging of a liquid by a moving plate, Acta Physicochim. USSR 17, 42 (1942).

[15] J. Seiwert, C. Clanet, and D. Quéré, Coating of a textured solid, J. Fluid Mech. 669, 55 (2011).

[16] R. Clift, J. R. Grace, and M. E. Weber, Bubbles, Drops and Particles (Academic Press, New York, 1978).

[17] D. Legendre, R. Zenit, and J. R. Velez-Cordero, On the deformation of gas bubbles in liquids, Phys. Fluids 24, 043303 (2012).
[18] J. Zawala, M. Krasowska, T. Dabros, and K. Malysa, Influence of bubble kinetic energy on its bouncing during collisions with various interfaces, Can. J. Chem. Eng. 85, 669 (2007).

[19] R. Zenit and D. Legendre, The coefficient of restitution for air bubbles colliding against solid walls in viscous liquids, Phys. Fluids 21, 083306 (2009).

[20] J. Zawala and K. Malysa, Influence of the impact velocity and size of the film formed on bubble coalescence time at water surface, Langmuir 27, 2250 (2011).

[21] R. Manica, E. Klaseboer, and D. Chan, Force balance model for bubble rise, impact, and bounce from solid surfaces, Langmuir 31, 6763 (2015).

[22] G. I. Taylor, The dynamics of thin sheets of fluid III. Disintegration of fluid sheets, Proc. R. Soc. A 253, 313 (1959).

[23] F. E. C. Culick, Comments on a ruptured soap film, J. Appl. Phys. 31, 1128 (1960).

[24] See Supplemental Material at http://link.aps.org/ supplemental/10.1103/PhysRevLett.117.094501 for additional experiments, concerning in particular the case of partial wetting.

[25] J. D. Paulsen, R. Carmigniani, A. Kannan, J. C. Burton, and S. R. Nagel, Coalescence of bubbles and drops in an outer fluid, Nat. Commun. 5, 3182 (2014).

[26] J. P. Munro, C. R. Anthony, O. A. Basaran, and J. R. Lister, Thin-sheet flow between coalescing bubbles, J. Fluid Mech. 773, R3 (2015). 